\title{
ANÁLISE SITUACIONAL DA APLICAÇÃO DAS BOAS PRÁTICAS DE FABRICAÇÃO NOS RESTAURANTES DO MUNICÍPIO DE CURRAIS NOVOS/RN
}

\author{
Nísia Luiza de Andrade Oliveira \\ Pesquisas Aplicadas em Ciência e Tecnologia de Alimentos, Instituto Federal de Educação, \\ Ciência e Tecnologia, Currais Novos, Estudante do IFRN, do curso técnico de Integrado em \\ Alimentos.e-mail: nisiaandrade@ @otmail.com \\ Odisséia Carla Pires Gaspareto \\ Pesquisas Aplicadas em Ciência e Tecnologia de Alimentos, IFRN, Campus Currais Novos, \\ Professora do curso Técnico em Alimentos. \\ odisseia.gaspareto@ifrn.edu.br
}

\section{RESUMO}

Nos últimos tempos tem-se obervado uma grande preocupação com o manejo adequado dos alimentos (desde a produção e manipulação da matéria-prima, até o processamento, distribuição e comercialização do produto acabado). Esta preocupação, deve-se à prevenção das doenças ocasionadas pela ingestão de alimentos contaminados, sendo a forma mais adequada para alcançá-la a utilização de métodos higiênicos. O interesse dos governos (mundiais, federais, estaduais, dentre outros) por este assunto vem sendo demonstrado através dos anos, principalmente, na elaboração de legislações voltadas para o controle higiênicosanitário dos locais de produção e comércio dos alimentos. Tendo como base estas características, este projeto tem como objetivo avaliar a aplicação das Boas Práticas de Fabricação nos restaurantes do município de Currais Novos, através do emprego de um questionário (check-list) dividido em 6 categorias: higiene dos funcionários, higiene dos utensílios e equipamentos, higiene dos alimentos, planejamento físico e funcional e higiene da área de refeição. As perguntas podem ser respondidas em SIM, NÃO ou NÃO SE APLICA, as quais constam com pontuação de 2,1 e 0 pontos, respectivamente. De acordo com os resultados do check-list pode-se concluir quais são os restaurantes que necessitam de um maior conhecimento da legislação (RDC Resolução no 216 de 15 de setembro de 2004).

PALAVRAS-CHAVE: Boas Práticas de Fabricação, questionário (check-list) e legislação.

\section{SITUATIONAL ANALYSIS OF APLICATION OF THE GOOD MANUFACTURING PRACTICE IN THE RESTAURANTS OF CURRAIS NOVOS/RN}

\begin{abstract}
Lately it's been possible to observe a great concernment with the adequate handling of food (since the production and handling of the raw material, until the processing step, distribution and selling of the finished product). This concernment is due to the prevention of the diseases caused by the ingestion of contaminated food, when the most adequate way of preventing this is the use of hygienic methods. The government interests (worldwide, federal, state and others) for this subject have been demonstrated through the last years especially with the elaboration of laws centred in the hygienic-sanitary control of the production places and commerce of food. Taking into consideration these characteristics, this project aims to evaluate the application of Good manufacturing practice in the restaurants of Currais Novos
\end{abstract}


county, through the application of a questionnaire (check-list) divided into 6 categories: employees hygiene, hygiene of the utensils and equipments, hygiene of food, physical and functional planning and the hygiene of the eating area. The questions can be answered with YES, NO or IT DOESN'T APPLY, which vary from 2, 1 or 0 points respectively. According to the results of the check-list it is possible to observe what restaurants need a better knowledge of the law (RDC Resolution nr 216 of September $15^{\text {th }}, 2004$ ).

KEY-WORDS: Good manufacturing practice, questionnaire (Check-list) and law. 


\section{ANÁLISE SITUACIONAL DA APLICAÇÃO DAS BOAS PRÁTICAS DE FABRICAÇÃO NOS RESTAURANTES DO MUNICÍPIO DE CURRAIS NOVOS/RN}

\section{INTRODUÇÃO}

A segurança alimentar tem tomado grande importância ao longo do tempo. Afinal, a possibilidade de garantir um produto que ofereça o menor risco de contaminação ao mercado consumidor tem se tornado uma grande preocupação para o governo e para os órgãos fiscalizadores

Um dos métodos mais utilizados para evitar riscos aos alimentos é a aplicação das Boas Práticas de Fabricação - BPF's - (BRASIL. RESOLUÇÃO RDC No 216, 2004), que são procedimentos que têm o intuito de estabelecer métodos de higiene afim de evitar a contaminação aos produtos. As quais, devem ser adotadas por estabelecimentos de produção e comercialização de alimentos. Vale ressaltar, que as boas práticas são regidas por legislações. As quais possuem maior ênfase nas BPF's são a Resolução RDC n 216/2004 e a Portaria $n^{\circ}$ 326/1997, ambas do Ministérios da Saúde.

Para identificar os estabelecimentos que utilizam as Boas Práticas de Fabricação, é necessário haver a inspeção destes locais, esta é realizada por alguns órgãos (Ministério da Saúde e Ministério da Agricultura, Pecuária e Abastecimento). No caso do Brasil, o principal órgão responsável para efetuar a fiscalização e a regulamentação dessas casas de produção é a Agência Nacional de Vigilância Sanitária (ANVISA), que está diretamente ligada ao Ministério da Saúde e tem como objetivo, "focar os regulamentos no aspecto de risco à saúde do cosumidor, apoiar a descentralização das ações e responsabilizar os diversos atores da cadeia produtiva" (BASTOS, 2008).

A partir da análise desses aspectos e tendo em vista um olhar crítico - que pode ser adquirido durante o curso de técnico em Alimentos - das condições dos restaurantes do município de Currais Novos, fez-se necessário a elaboração de um projeto que abordasse o acesso ao conhecimento dos proprietários, funcionários ou técnicos desses estabelecimentos sobre a legislação e sobre as Boas Práticas de Fabricação, desta forma, será constatado como se encontra o campo de informação desses indivíduos e se esses locais propiciam um alimento seguro para os seus consumidores.

Na elaboração do projeto foi utilizada uma das legislações que trata sobre Boas Práticas de Fabricação, a RDC Resolução 216 de 15 de setembro de 2004, ela tem como objetivo "estabelecer procedimentos de boas práticas para serviços de alimentação, a fim de garantir as condições higiênico-sanitárias do alimento preparado" (BRASIL. RESOLUÇÃO RDC N 216 , 2004). Portanto, a qual serviu como base para todos os procedimentos deste projeto, desde a elaboração do check-list, até à conclusão deste.

\section{MATERIAIS E MÉTODOS}

Primeiramente foi feita uma pesquisa aprofundada de projetos que seguiam este mesmo objetivo - análise das BPF em estabelecimentos de produção e venda de alimentos - e uma leitura minunciosa da Resolução RDC n²16/2004, com intuito de aprimorar os conhecimentos sobre os métodos utilizados nas outras pesquisas sobre a legislação.

Após a obtenção das informações iniciou-se a elaboração do questionário (check-list),o qual era dividido em 6 (seis) categorias que continham perguntas que se enquadravam diretamente 
com elas: higiene dos funcionários (30 perguntas), higiene dos utensílios e equipamentos (13 perguntas), higiene da cozinha (20 perguntas), higiene dos alimentos (41 perguntas), planejamento físico funcional (44 perguntas) e higiene do refeitório (4 perguntas). As perguntas só poderiam ser respondidas em $\operatorname{SIM}(\mathrm{S})$, NÃO(N) E NÃO SE APLICA (NA), as quais possuíam 2,1 e 0 pontos, respectivamente.

Com o check-list elaborado foi realizada a seleção dos restaurantes, que ocorreu da seguinte forma: escolhemos aleatoriamente 10 (dez) estabelecimentos do município, em seguida, sorteamos 6 (seis), nos quais foi aplicado o check-list com os proprietários (em 4 dos restaurantes) ou com os responsáveis/técnico (em 2 dos restaurantes).

\section{RESULTADOS E DISCUSSÕES}

Após a aplicação do questionário foram avaliadas as conformidades e não conformidades de cada restaurante, de acordo com as categorias abordadas no mesmo. Com os resultados obtidos através do quetionário, foram feitos gráficos de cada quisito do check-list para facilitar a compreensão da análise.

Na categoria Higiene dos funcionários (Figura 1), todos os pontos de comércio alcançaram mais de 60\% (sessenta por cento) das conformidades. O que demonstra que os estabelecimentos prezam pela adequação dos seus colaboradores. A mesma porcentagem foi alcançada na Higiene dos utensílios e equipamentos (Figura 2), evidenciando que os equipamentos e utensílios destinados à produção estão sendo higienizados.

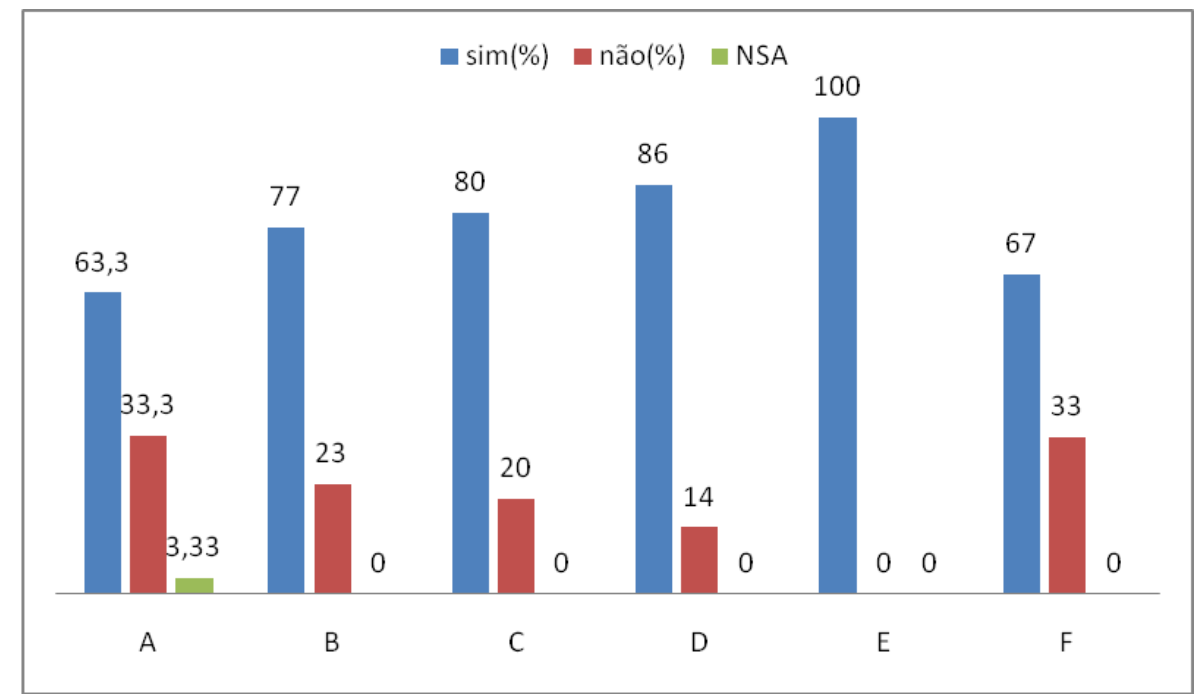

Figura 1 - Higiene dos funcionários.

Nos quesitos Higiene da cozinha (Figura 3) e Higiene dos alimentos (Figura 4), os restaurantes tiveram no mínimo $70 \%$ (setenta por cento) de conformidade. Desta forma, é possível constatar que o ambiente da produção e, tanto a matéria-prima, como o produto acabado estão seguindo as normas e garantindo a segurança dos consumidores.

O Planejamento físico funcional ( Figrua 5), obteve, no mínimo, 50\% das conformidades, o que deixa a desejar, já que está categoria é de suma importância para evitar, principalmente, a contaminação cruzada, como o afastamente entre os banheiros e a área de produção, dentre outras. 


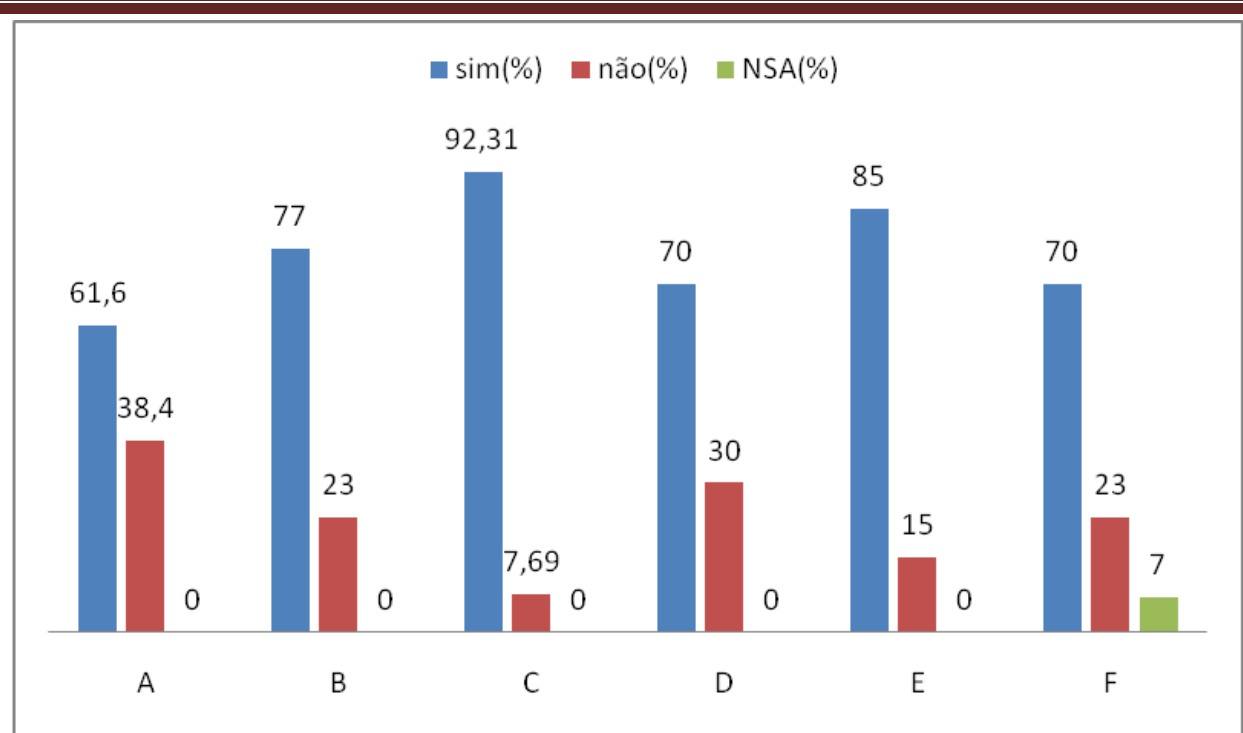

Figura 2 - Higiene dos utensílios e equipamentos

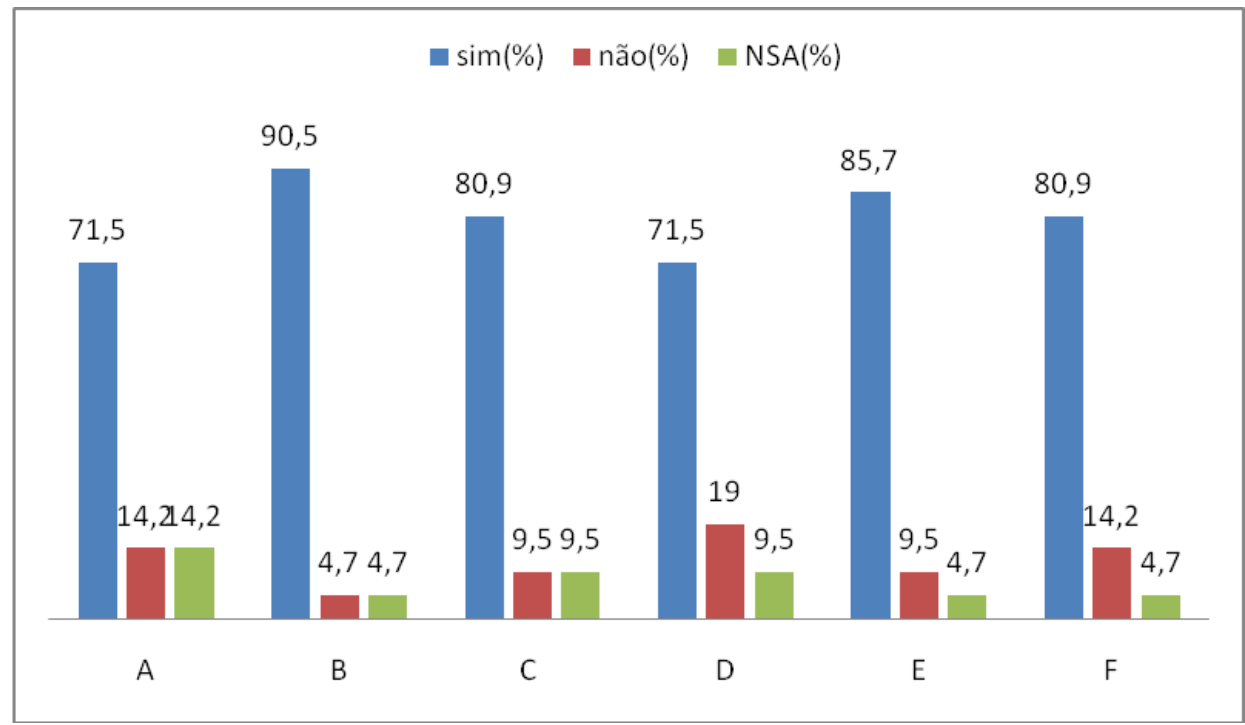

Figura 3 - Higiene da cozinha

No que concerne a última categoria, Higiene do refeitório (Figura 6), alcançou, no mínimo, $75 \%$ de conformidade. Através desse resultado, pode-se observar que esta área é o local que os consumidores mais tem acesso, e por isso, é a que mais segue as exigências da RDC n²16/2004.

Em seguida, com todos os gráficos efetuados, foi feita uma média aritmética com os resultados das categorias de cada restaurante (Figura 7), desta forma, conclui-se quais são os restaurantes que mais se enquadraram e quais necessitam de maior informações e mudanças para se adequar aos padrões exigidos pela legislação. 


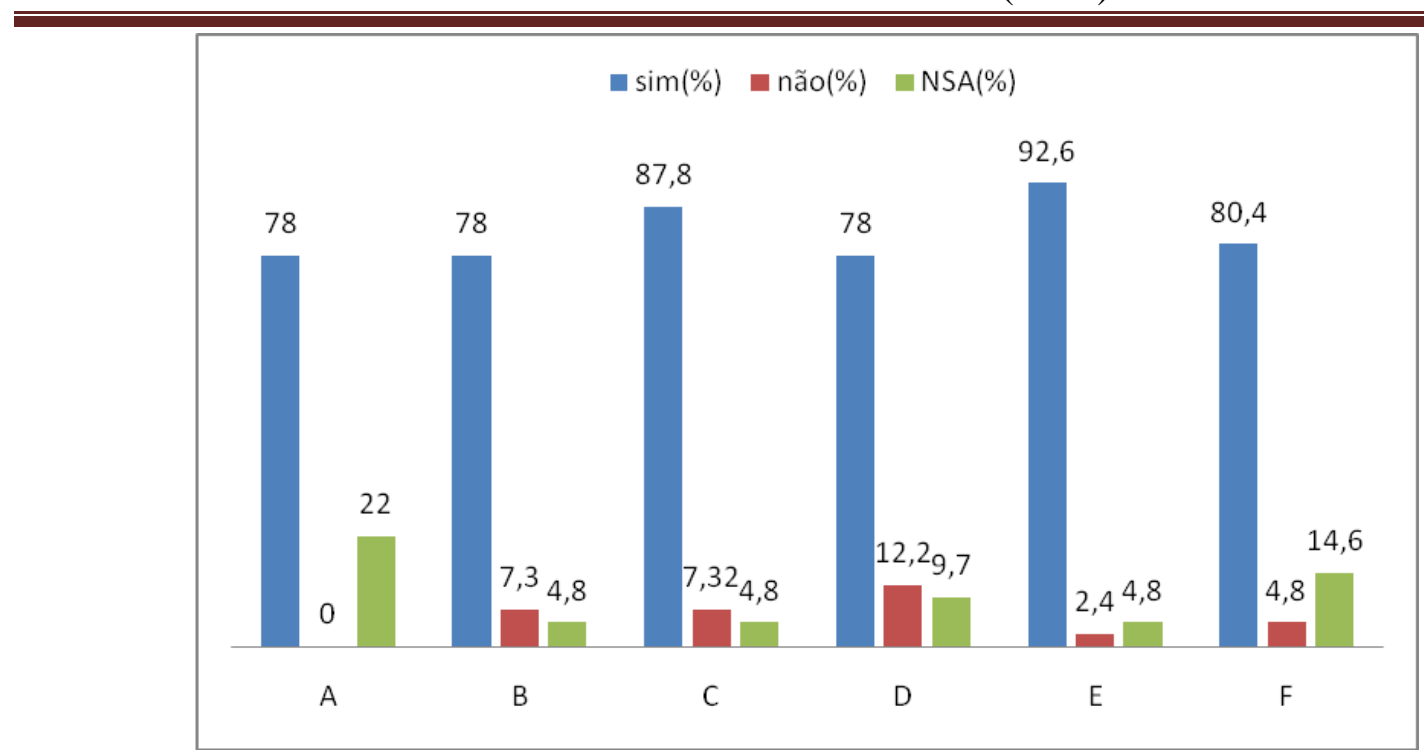

Figura 4 - Higiene dos alimentos

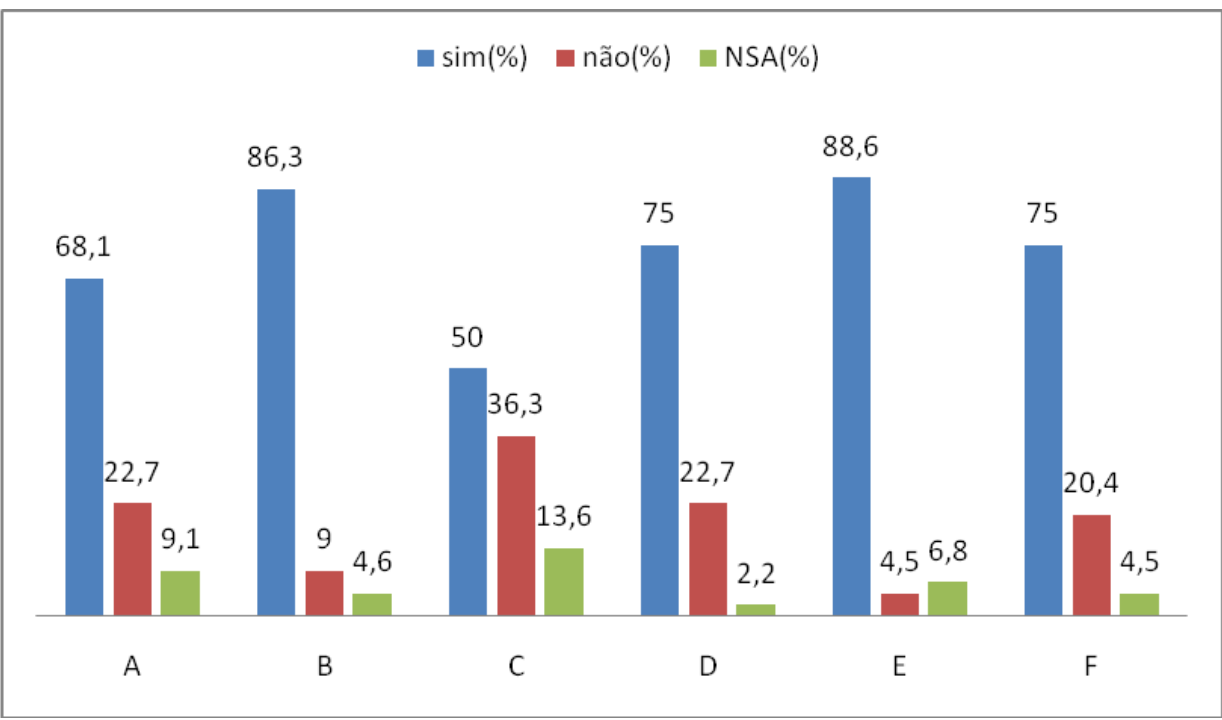

Figura 5 - Planejamento físico funcional

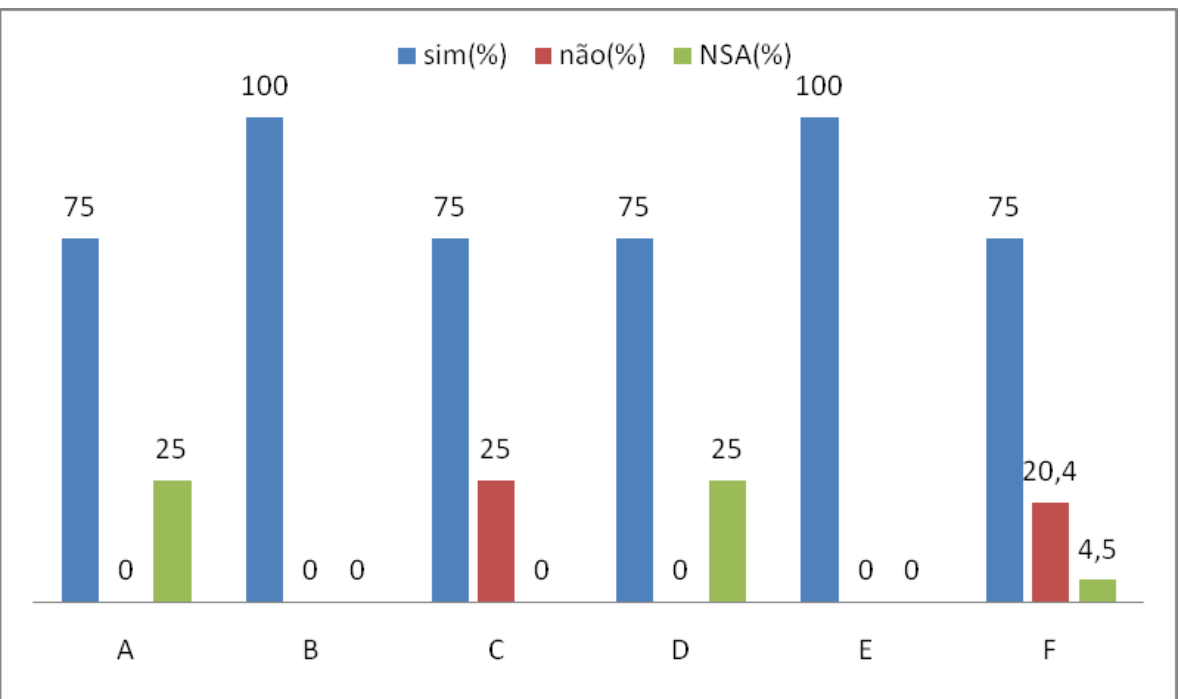

Figura 6 - Higiene do Refeitório 


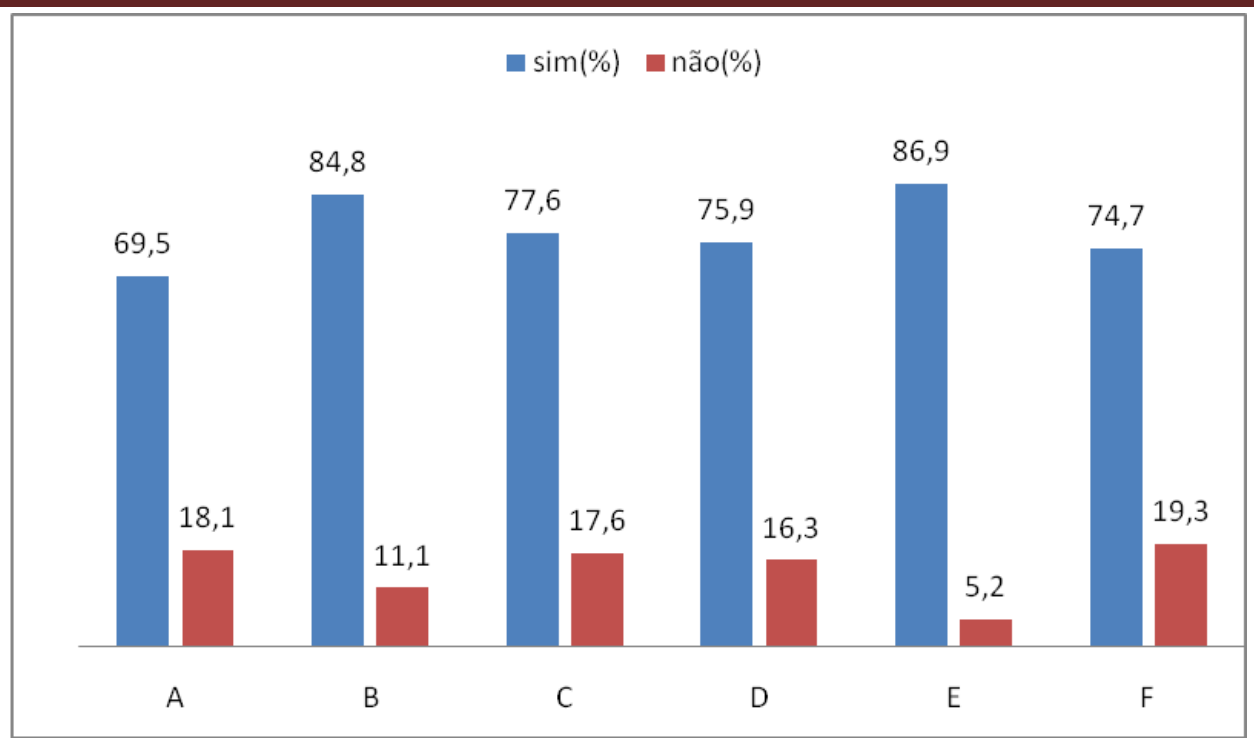

Figura 7 - Médias aritméticas das categorias

\section{CONCLUSÃO}

Após a análise das Médias aritméticas das categorias de cada Restaurante, concluimos que os Restaurantes $\boldsymbol{E}$ alcançou $86,9 \%$ das conformidades, e o Restaurante $\boldsymbol{A}$ foi o que obteve menores adequações alcançando apenas $69,5 \%$ das conformidades. Vale salientar que, apesar dos resultados obtidos no Restaurante $\boldsymbol{E}$ este precisa de modificações, visto que o resultado mínimo para garantir a a segurança mínima do alimento é de $90 \%$.

\section{REFERÊNCIAS}

1. BRASIL. RESOLUÇÃO RDC No 216, 2004.

2. GERMANO, Pedro Manuel Leal \& GERMANO, Maria Izabel Simões Germano. Higiene e vigilância sanitária de alimentos - 3. ed. rev. e ampl.- Barueri, SP: Manole Ltda, 2008.

3. MALINVERNO, Emanoele; FRANCISCO, Diane Carina; ROZA, Cleber Rabelo. Verificação da Implantação das Boas Práticas de Fabricação em Restaurantes de Farroupilha, RS. Revista Higiene Alimentar, São Paulo, v. 23, n.178/179, p. 36-38, nov./dez. 2009.

4. QUINTILIANO et al. Avaliação das condições higiênico-sanitária em restaurantes, com Aplicação de Ficha de Inspeção Baseada na Legislação Federal, RDC 216/2004. Revista Higiene Alimentar, São Paulo, v.22, n. 160, p. 25-30, abr. 2008.

5. REOLON, Carla Aparecida; SILVA, Sebastião Marculino. Condições Higiênicosanitárias de Restaurantes do Município de Medianeira, PR. Revista Higiene Alimentar, São Paulo, v.23, n. 174/175, p. 55-56, jul./ago. 2009.

6. SOUTHIER, Naiara; NOVELLO, Daiana. Treinamento, Avaliação e Orientação de Manipuladores, Sobre Boas Práticas de Higine em uma Unidade de Alimentação e Nutrição da Cidade de Guarapuava, PR. Revista Higiene Alimentar, São Paulo, v. 22, n. 162, p. 45-50, jun. 2008.

7. STANGARLIN et al. Avaliação da Implementação do Manual de Boas Práticas e Procedimentos Operacionais Padronizados em serviços de Alimentação $2^{\mathbf{a}}$ parte. Revista Higiene Alimentar, São Paulo, v. 23,n. 168/169, p. 24-27, jan./fev. 2009. 
8. BASTOS, Maria do Socorro Rocha. Ferramentas da ciência e tecnologia para a segurança dos alimentos. 2 ed.- Fortaleza, CE. Ed. Moderna, 2008. 